PROCEEDINGS OF THE

AMERICAN MATHEMATICAL SOCIETY

Volume 134, Number 10, October 2006, Pages 3085-3095

S 0002-9939(06)08334-1

Article electronically published on May 5, 2006

\title{
OPEN SUBGROUPS AND THE CENTRE PROBLEM FOR THE FOURIER ALGEBRA
}

\author{
ZHIGUO HU
}

(Communicated by N. Tomczak-Jaegermann)

\begin{abstract}
Let $A(G)$ be the Fourier algebra of a locally compact group and $U C B(\hat{G})$ the $C^{*}$-algebra of uniformly continuous linear functionals on $A(G)$. We study how the centre problem for the algebra $U C B(\hat{G})^{*}$ (resp. $A(G)^{* *}$ ) is related to the centre problem for the algebras $U C B(\hat{H})^{*}$ (resp. $A(H)^{* *}$ ) of $\sigma$-compact open subgroups $H$ of $G$. We extend some results of Lau-Losert on the centres of $U C B(\hat{G})^{*}$ and $A(G)^{* *}$.
\end{abstract}

\section{INTRODUCTION}

Let $A$ be a Banach algebra. As is well known, there exist two Banach algebra multiplications on the second dual $A^{* *}$ of $A$ such that each of them extends the multiplication on $A$ (cf. Arens [1]). We will always consider the first Arens multiplication on $A^{* *}$ throughout this paper. The dual of the space $\overline{\operatorname{span}}\left(A^{*} A\right)$ equipped with the multiplication induced by that on $A^{* *}$ is also a Banach algebra. In recent years, the topological centre problem for the algebras $A^{* *}$ and $\left[\overline{\operatorname{span}}\left(A^{*} A\right)\right]^{*}$, in particular for $A$ being some Banach algebras associated with a locally compact group, has attracted some attention. Let $A$ be either the group algebra $L^{1}(G)$ or the Fourier algebra $A(G)$ of a locally compact group $G$. Then the corresponding algebras $\left[\overline{\operatorname{span}}\left(A^{*} A\right)\right]^{*}$ are $L U C(G)^{*}$ and $U C B(\hat{G})^{*}$, respectively, where $L U C(G)$ is the space of bounded left uniformly continuous functions on $G$ and $U C B(\hat{G})$ is the space of uniformly continuous linear functionals on $A(G)$.

Let $Z_{t}\left(A^{* *}\right)$ (resp. $\left.Z_{t}\left(\left[\overline{\operatorname{span}}\left(A^{*} A\right)\right]^{*}\right)\right)$ be the topological centre of $A^{* *}$ (resp. $\left.\left(\overline{\operatorname{span}}\left(A^{*} A\right)\right)^{*}\right)$. In [5], Grosser-Losert showed that $Z_{t}\left(L U C(G)^{*}\right)=M(G)$ if $G$ is abelian, where $M(G)$ is the measure algebra of $G$. Lau [15] extended this result to all locally compact groups. For the group algebra $L^{1}(G)$, Isik-Pym-Ülger [12] proved that if $G$ is compact, then $Z_{t}\left(L^{1}(G)^{* *}\right)=L^{1}(G)$. This result has also been extended to all locally compact groups by Lau-Losert [16.

When $G$ is abelian with dual group $\Gamma, L^{1}(\Gamma) \cong A(G), L U C(\Gamma) \cong U C B(\hat{G})$ and $M(\Gamma) \cong B_{\rho}(G)$ (the reduced Fourier-Stieltjes algebra of $G$ ). Therefore, if $G$ is abelian, then $Z\left(U C B(\hat{G})^{*}\right)=B_{\rho}(G)$ and $Z\left(A(G)^{* *}\right)=A(G)$. It is natural to consider when the above equalities hold for a non-abelian locally compact group.

Received by the editors January 11, 2005 and, in revised form, May 5, 2005.

2000 Mathematics Subject Classification. Primary 22D25, 43A30.

Key words and phrases. Fourier algebra, reduced Fourier-Stieltjes algebra.

This research was supported by an NSERC grant.

(C)2006 American Mathematical Society Reverts to public domain 28 years from publication 
Lau-Losert [17 showed that if $G$ is second countable and $\overline{[G, G]}$ is not open in $G$, where $[G, G]$ denotes the commutator subgroup of $G$, then

(i) $Z_{t}\left(U C B(\hat{G})^{*}\right)=B_{\rho}(G)$;

(ii) $Z_{t}\left(A(G)^{* *}\right)=A(G)$ if $G$ is assumed to be amenable.

Clearly, (i) holds for all discrete groups (cf. [14, Proposition 4.5] and [17, Theorem 5.8]). Lau-Losert [17, Theorem 6.5(i)] proved that $Z_{t}\left(A(G)^{* *}\right)=A(G)$ is also true when $G$ is discrete and amenable. Moreover, Lau-Losert [18] proved that (i) and (ii) hold if $G$ is a countably infinite product of second countable locally compact groups $\left\{G_{i}\right\}_{i=0}^{\infty}$ with $G_{i}(i \geq 1)$ compact and non-trivial. However, a special consequence of Losert [19, Theorem 3] says that $A(G) \neq Z_{t}\left(A(G)^{* *}\right)\left(\neq A(G)^{* *}\right)$ if $G$ is a discrete group containing the free group $F_{r}$ on $r$ generators $(2 \leq r<\infty)$. Very recently, Losert further showed that (ii) fails for $G=S U(3)$.

In this paper, we are concerned with locally compact groups with a large compact covering number. We study how the topological centre problem (i) for $U C B(\hat{G})^{*}$ is related to the same problem for the algebras $U C B(\hat{H})^{*}$ of open subgroups $H$ of $G$. We prove that $Z_{t}\left(U C B(\hat{G})^{*}\right)=B_{\rho}(G)$ if and only if $Z_{t}\left(U C B(\hat{H})^{*}\right)=B_{\rho}(H)$ for all $\sigma$-compact open subgroups $H$ of $G$ (Theorem 3.4). We further investigate whether the parallel result holds for $A(G)^{* *}$ (cf. Theorem 3.9). As an application, we extend some results of Lau-Losert on $Z_{t}\left(U C B(\hat{G})^{*}\right)$ and $Z_{t}\left(A(G)^{* *}\right)$ to metrizable locally compact groups (cf. Theorem 3.14 and Theorem 3.16).

\section{Preliminaries}

Let $A$ be a Banach algebra. Then $A^{*}$ is a Banach $A$-bimodule under the actions

$$
\langle x \cdot \phi, \psi\rangle=\langle x, \phi \psi\rangle \quad \text { and } \quad\langle\phi \cdot x, \psi\rangle=\langle x, \psi \phi\rangle \quad\left(x \in A^{*} \text { and } \phi, \psi \in A\right) .
$$

Each of these two module actions naturally induces a Banach algebra multiplication on $A^{* *}$ which extends that on $A$ (cf. Arens [1]). Let · and $\triangle$ denote the first and the second Arens multiplications on $A^{* *}$, respectively. Evidently, for any fixed $m \in A^{* *}$, the maps $n \mapsto n \cdot m$ and $n \mapsto m \triangle n$ are weak*-weak* continuous on $A^{* *}$. The first and the second topological centres of $A^{* *}$ are defined as follows:

$$
\begin{aligned}
& Z_{t}^{1}\left(A^{* *}\right)=\left\{m \in A^{* *}: \text { the map } n \mapsto m \cdot n \text { is } w^{*}-w^{*} \text { continuous on } A^{* *}\right\}, \\
& Z_{t}^{2}\left(A^{* *}\right)=\left\{m \in A^{* *}: \text { the map } n \mapsto n \triangle m \text { is } w^{*}-w^{*} \text { continuous on } A^{* *}\right\} .
\end{aligned}
$$

It is readily seen that $A \subseteq Z_{t}^{1}\left(A^{* *}\right) \cap Z_{t}^{2}\left(A^{* *}\right)$. $A$ is said to be Arens regular if $Z_{t}^{1}\left(A^{* *}\right)=Z_{t}^{2}\left(A^{* *}\right)=A^{* *}$.

Let $X$ be a topologically left invariant subspace of $A^{*}$ (i.e., $X \cdot A \subseteq X$ ). For $m \in X^{*}$ and $x \in X$, one can define $m \cdot x \in A^{*}$ by

$$
\langle m \cdot x, \phi\rangle=\langle m, x \cdot \phi\rangle \quad(\phi \in A) .
$$

$X$ is called topologically left introverted if $m \cdot x \in X$ for all $m \in X^{*}$ and $x \in X$. It can be seen that $\overline{\operatorname{span}}\left(A^{*} A\right)$ is topologically left introverted in $A^{*}$ (cf. the proof of Lau [14, Proposition 5.2]).

Let $X$ be a topologically left introverted subspace of $A^{*}$. Then $X^{*}$ becomes a Banach algebra under the multiplication defined by $\langle m \cdot n, x\rangle=\langle m, n \cdot x\rangle \quad(m, n \in$ $X^{*}$ and $\left.x \in X\right)$. It is evident that this multiplication on $X^{*}$ is induced by the first Arens multiplication on $A^{* *}$. That is, if $m, n \in X^{*}$ and $\tilde{m}, \tilde{n} \in A^{* *}$ are extensions of $m, n$, respectively, then $\tilde{m} \cdot \tilde{n} \in A^{* *}$ is an extension of $m \cdot n$. Obviously, for any 
fixed $m \in X^{*}$, the map $n \mapsto n \cdot m$ is weak*-weak* continuous on $X^{*}$. The (left) topological centre of $X^{*}$ is defined as

$$
Z_{t}\left(X^{*}\right)=\left\{m \in X^{*}: \text { the map } n \mapsto m \cdot n \text { is } w^{*}-w^{*} \text { continuous on } X^{*}\right\} \text {. }
$$

If $A$ is a commutative Banach algebra, then $Z_{t}^{1}\left(A^{* *}\right)=Z_{t}^{2}\left(A^{* *}\right)$ is just the algebraic centre $Z\left(A^{* *}\right)$ of $A^{* *}$ (equipped with either of the Arens multiplications). The following lemma is clearly true.

Lemma 2.1. Let $A$ be a commutative Banach algebra and let $X$ be a topologically introverted subspace of $A^{*}$. Then $Z_{t}\left(X^{*}\right)$ is the algebraic centre $Z\left(X^{*}\right)$ of $X^{*}$.

For a linear subspace $Y$ of $A^{*}$ containing $A^{*} A, y^{*} \in Y^{*}$ and $f \in A^{*}$, let $y^{*} \cdot f \in A^{*}$ be defined by $\left\langle y^{*} \cdot f, a\right\rangle=\left\langle y^{*}, f \cdot a\right\rangle(a \in A)$. If $X$ is a subset of $A^{*}, X^{\perp}$ will denote the annihilator of $X$ in $A^{* *}$. Here we collect some simple facts on $A^{*} A$ and $Z\left(A^{* *}\right)$, which will be used in the sequel.

Lemma 2.2. Let $A$ be a commutative Banach algebra. Then

(i) $\left(A^{*} A\right)^{\perp}=\left\{m \in A^{* *}: n \cdot m=0\right.$ for all $\left.n \in A^{* *}\right\}$.

(ii) $\left(A^{* *} A^{*}\right)^{\perp}=\left\{m \in A^{* *}: m \cdot n=n \cdot m=0\right.$ for all $\left.n \in A^{* *}\right\}$.

(iii) $Z\left(A^{* *}\right) \cap\left(A^{*} A\right)^{\perp}=\left(A^{* *} A^{*}\right)^{\perp}$.

(iv) $\left[\overline{\operatorname{span}}\left(A^{*} A\right)\right]^{*} \cdot A^{*}=A^{* *} A^{*}$ and $Z\left(A^{* *}\right) \cap\left(A^{*} A\right)^{\perp}=\left(\left[\overline{\operatorname{span}}\left(A^{*} A\right)\right]^{*} \cdot A^{*}\right)^{\perp}$.

Proof. (i) is obviously true, and (ii) is included in [19, Lemma 1]. By (ii), we have $\left(A^{* *} A^{*}\right)^{\perp} \subseteq Z\left(A^{* *}\right)$. Therefore, (iii) follows from (i) and (ii).

For (iv), let $y^{*} \in\left[\overline{\operatorname{span}}\left(A^{*} A\right)\right]^{*}$. It is easy to see that if $n \in A^{* *}$ is an extension of $y^{*}$, then $y^{*} \cdot f=n \cdot f$ for all $f \in A^{*}$. Therefore, $\left[\overline{\operatorname{span}}\left(A^{*} A\right)\right]^{*} \cdot A^{*}=A^{* *} A^{*}$ and hence, by (iii), $Z\left(A^{* *}\right) \cap\left(A^{*} A\right)^{\perp}=\left(A^{* *} A^{*}\right)^{\perp}=\left(\left[\overline{\operatorname{span}}\left(A^{*} A\right)\right]^{*} \cdot A^{*}\right)^{\perp}$.

Let $G$ be a locally compact group. The Fourier-Stieltjes algebra $B(G)$ is the linear span of positive definite continuous functions on $G$ and can be identified with the dual of the group $C^{*}$-algebra $C^{*}(G)$ of $G$. With the dual norm and the pointwise multiplication, $B(G)$ is a commutative Banach algebra. The reduced Fourier-Stieltjes algebra $B_{\rho}(G)$ is the closure of $B(G) \cap C_{00}(G)$ in the $w^{*}$-topology of $B(G)$, where $C_{00}(G)$ is the set of continuous functions on $G$ with compact support. $B_{\rho}(G)$ is a closed ideal in $B(G)$ and is precisely the dual of the reduced group $C^{*}$-algebra $C_{\rho}^{*}(G)$ of $G$. As is known, $B_{\rho}(G)=B(G)$ if and only if $G$ is amenable.

The Fourier algebra $A(G)$ is the closed ideal in $B(G)$ generated by $B(G) \cap C_{00}(G)$. $A(G)$ can be identified with the predual of the group von Neumann algebra $V N(G)$ of $G$. Naturally, $V N(G)$ is a Banach $B(G)$-module under the action defined by $\langle u \cdot T, v\rangle=\langle T, u v\rangle(u \in B(G), v \in A(G)$ and $T \in V N(G))$. See Eymard [2] for more information on $B(G), B_{\rho}(G), A(G)$, and $V N(G)$.

The support of an operator $T$ in $V N(G)$ is defined by saying that $x \in \operatorname{supp} T$ if and only if $u \cdot T=0$ implies $u(x)=0$ for all $u \in A(G)$ (cf. Eymard [2] and Herz [6]). The space $U C B(\hat{G})$ of uniformly continuous linear functionals on $A(G)$ is the norm closure of $A(G) \cdot V N(G)$ in $V N(G)$. It is known that $U C B(\hat{G})$ is a $C^{*}$-subalgebra of $V N(G)$ and also a closed $B(G)$-submodule of $V N(G)$ which coincides with the norm closure of $\{T \in V N(G): \operatorname{supp} T$ is compact $\}$ in $V N(G)$ (cf. Granirer [3]- 4]).

We recall that $U C B(\hat{G})$ is a topologically introverted subspace of $V N(G)$. Thus, $U C B(\hat{G})^{*}$ is a Banach algebra and $Z_{t}\left(U C B(\hat{G})^{*}\right)$ is just the algebraic centre $Z\left(U C B(\hat{G})^{*}\right)$ of $U C B(\hat{G})^{*}$ (cf. Lemma 2.1). 
Throughout this paper, $H$ will denote an open subgroup of $G$. Let $r_{H}: A(G) \rightarrow$ $A(H)$ be the restriction map and $t_{H}: A(H) \rightarrow A(G)$ the trivial extension map (i.e., $\left(t_{H} u\right)(x)=0$ for $\left.x \in G-H\right)$. The adjoint map $r_{H}^{*}$ is a $*$-isomorphism of $V N(H)$ onto the sub von Neumann algebra $V N_{H}(G)$ of $V N(G)$, where

$$
V N_{H}(G)=\{T \in V N(G): \operatorname{supp} T \subseteq H\}
$$

(cf. Eymard [2, Proposition 3.21]). Also, $r_{H}^{* *}$ is an algebraic homomorphism of $A(G)^{* *}$ onto $A(H)^{* *}$ and $t_{H}^{* *}$ is an algebraic isomorphism of $A(H)^{* *}$ into $A(G)^{* *}$.

It is known that $r_{H}^{*}(U C B(\hat{H})) \subseteq U C B(\hat{G})$ and $t_{H}^{*}(U C B(\hat{G}))=U C B(\hat{H})$ (cf. Granirer [3]). We let $\Phi=\left.r_{H}^{*}\right|_{U C B(\hat{H})}: U C B(\hat{H}) \rightarrow U C B(\hat{G})$ and $\Psi=\left.t_{H}^{*}\right|_{U C B(\hat{G})}$ : $U C B(\hat{G}) \rightarrow U C B(\hat{H})$. Then $\Psi \circ \Phi=i d$ and $\Phi$ is a $*$-isomorphism of $U C B(\hat{H})$ onto $U C B(\hat{G}) \cap V N_{H}(G)$. Furthermore, $\Phi^{*}$ is an algebraic homomorphism of $U C B(\hat{G})^{*}$ onto $U C B(\hat{H})^{*}$, and $\Psi^{*}$ is an algebraic isomorphism of $U C B(\hat{H})^{*}$ into $U C B(\hat{G})^{*}$.

A direct computation shows the following result on the images of the centres under the maps $\Phi^{*}$ and $\Psi^{*}$.

Lemma 2.3. Let $G$ be a locally compact group and let $H$ be an open subgroup of G. Then

(i) $\Psi^{*}\left[Z\left(U C B(\hat{H})^{*}\right)\right] \subseteq Z\left(U C B(\hat{G})^{*}\right)$.

(ii) $\Phi^{*}\left[Z\left(U C B(\hat{G})^{*}\right)\right]=Z\left(U C B(\hat{H})^{*}\right)$.

\section{The centres of $U C B(\hat{G})^{*}$ And $A(G)^{* *}$}

Let $G$ be a locally compact group. In [17, Lau-Losert defined an isometric embedding $\pi=\pi_{G}: B_{\rho}(G) \hookrightarrow U C B(\hat{G})^{*}$ satisfying

$$
\langle\pi(\varphi), u \cdot T\rangle=\langle T, \varphi u\rangle \quad \text { for } \varphi \in B_{\rho}(G), u \in A(G) \text { and } T \in V N(G),
$$

i.e., $\pi$ is the natural extension of the isometric embedding of $A(G)$ into $\operatorname{UCB}(\hat{G})^{*}$ (see Lau 14] for the amenable case). $\pi: B_{\rho}(G) \rightarrow U C B(\hat{G})^{*}$ is also an algebraic isomorphism, i.e., $\pi(\varphi \psi)=\pi(\varphi) \cdot \pi(\psi)\left(\varphi, \psi \in B_{\rho}(G)\right)$. Furthermore,

$$
\varphi \cdot T=\pi(\varphi) \cdot T \text { for all } \varphi \in B_{\rho}(G) \text { and } T \in V N(G),
$$

where $\varphi \cdot T$ is the $B_{\rho}(G)$-module product and $\pi(\varphi) \cdot T$ is as defined in Section 2 . In the following, $B_{\rho}(G)$ will be identified with the closed subalgebra $\pi\left(B_{\rho}(G)\right)$ of $U C B(\hat{G})^{*}$. Lau-Losert [17, Proposition 4.5] showed that $B_{\rho}(G) \subseteq Z\left(U C B(\hat{G})^{*}\right)$.

Note that $A(G) \subseteq B_{\rho}(G) \subseteq U C B(\hat{G})^{*}$. Applying Lemma 2.2 to $A(G)$, we obviously have the following

Corollary 3.1. Let $G$ be a locally compact group. Then

(i) $\left[V N(G)^{*} V N(G)\right]^{\perp}=\left[U C B(\hat{G})^{*} \cdot V N(G)\right]^{\perp} \subseteq\left[B_{\rho}(G) \cdot V N(G)\right]^{\perp} \subseteq$ $U C B(\hat{G})^{\perp}$.

(ii) $\left[V N(G)^{*} V N(G)\right]^{\perp}=Z\left(A(G)^{* *}\right) \cap\left[B_{\rho}(G) \cdot V N(G)\right]^{\perp}=Z\left(A(G)^{* *}\right) \cap$ $U C B(\hat{G})^{\perp}$.

(iii) $B_{\rho}(G)=U C B(\hat{G})^{*}$ and $V N(G)^{*} V N(G)=B_{\rho}(G) \cdot V N(G)$ if $G$ is discrete.

Let $\mathcal{H}_{0}$ be the collection of all $\sigma$-compact open subgroups of $G$.

Lemma 3.2. Let $G$ be a locally compact group and let $\theta$ be a function on $G$ such that $\left.\theta\right|_{H} \in B_{\rho}(H)$ for all $H \in \mathcal{H}_{0}$. Then $\theta \in B_{\rho}(G)$. 
Proof. First, we observe that there exists a constant $M>0$ such that $\left\|\left.\theta\right|_{H}\right\|_{B_{\rho}(H)} \leq$ $M$ for all $H \in \mathcal{H}_{0}$. Otherwise, for each positive integer $n$, there exists an $H_{n} \in \mathcal{H}_{0}$ such that $\left\|\left.\theta\right|_{H_{n}}\right\|_{B_{\rho}\left(H_{n}\right)} \geq n$. Let $H$ be the subgroup of $G$ generated by $H_{n}(n=$ $1,2, \cdots)$. Then $H \in \mathcal{H}_{0}$ and $\left\|\left.\theta\right|_{H}\right\|_{B_{\rho}(H)} \geq\left\|\left.\theta\right|_{H} \cdot 1_{H_{n}}\right\|_{B_{\rho}(H)}=\left\|\left.\theta\right|_{H_{n}}\right\|_{B_{\rho}\left(H_{n}\right)} \geq n$ for all $n$, which is a contradiction.

Next, we show that $\theta \in B_{\rho}(G)$. Note that $G=\bigcup_{H \in \mathcal{H}_{0}} H$ and $\|\cdot\|_{\infty} \leq\|\cdot\|_{B_{\rho}}$. Thus, $\theta$ is a bounded continuous function on $G$. Let $f \in L^{1}(G)$. Then there exists an $H \in \mathcal{H}_{0}$ such that $f=0$ on $G-H$. So,

$$
\left|\int_{G} f(x) \theta(x) d x\right|=\left|\int_{H} f(x) \theta\right|_{H}(x) d x\left|\leq\left\|\left.\theta\right|_{H}\right\|_{B_{\rho}(H)}\left\|\left.f\right|_{H}\right\|_{C_{\rho}^{*}(H)} \leq M\|f\|_{C_{\rho}^{*}(G)} .\right.
$$

Therefore, $\sup \left\{\left|\int_{G} f(x) \theta(x) d x\right|: f \in L^{1}(G)\right.$ and $\left.\|f\|_{C_{\rho}^{*}(G)} \leq 1\right\} \leq M<\infty$. According to [2, Proposition 2.1], $\theta \in B_{\rho}(G)$.

For any open subgroup $H$ of $G$, let $\lambda_{H}$ be the left regular representation of $H$ and let $m_{H}$ denote $\Phi^{*}(m)$ for $m \in U C B(\hat{G})^{*}$, where $\Phi: U C B(\hat{H}) \rightarrow U C B(\hat{G})$ is the $*$-isomorphism as defined in Section 2. It is easy to see that $\Phi\left(\lambda_{H}(h)\right)=\lambda_{G}(h)$ for all $h \in H$. Also, for all $x \in G$ and $\varphi \in B_{\rho}(G)$, we have $\left\langle\pi(\varphi), \lambda_{G}(x)\right\rangle=\varphi(x)$.

Lemma 3.3. Let $G$ be a locally compact group and let $m \in U C B(\hat{G})^{*}$ be such that $m_{H} \in B_{\rho}(H)$ for all $H \in \mathcal{H}_{0}$. Then $m \in B_{\rho}(G)$.

Proof. By the assumption, for each $H \in \mathcal{H}_{0}$, there exists a $\theta_{H} \in B_{\rho}(H)$ such that $m_{H}=\pi_{H}\left(\theta_{H}\right)$, where $\pi_{H}: B_{\rho}(H) \hookrightarrow U C B(\hat{H})^{*}$ is the isometric embedding.

For $H \in \mathcal{H}_{0}$ and $x \in H$, we have

$$
\left\langle m, \lambda_{G}(x)\right\rangle=\left\langle m, \Phi\left(\lambda_{H}(x)\right)\right\rangle=\left\langle m_{H}, \lambda_{H}(x)\right\rangle=\left\langle\pi_{H}\left(\theta_{H}\right), \lambda_{H}(x)\right\rangle=\theta_{H}(x) .
$$

Thus, $\theta_{H}(x)=\left\langle m, \lambda_{G}(x)\right\rangle$ for all $H \in \mathcal{H}_{0}$ and $x \in H$. Let $\theta(x)=\left\langle m, \lambda_{G}(x)\right\rangle$ $(x \in G)$. Then $\left.\theta\right|_{H}=\theta_{H} \in B_{\rho}(H)$ for all $H \in \mathcal{H}_{0}$. By Lemma 3.2, $\theta \in B_{\rho}(G)$.

To show that $m=\pi(\theta)$, let $T \in U C B(\hat{G})$. Note that $\{R \in V N(G): \operatorname{supp} R$ is compact $\}$ is norm dense in $U C B(\hat{G})$. So, we assume that $\operatorname{supp} T$ is compact. Then $\operatorname{supp} T \subseteq H$ for some $H \in \mathcal{H}_{0}$, i.e., $T \in V N_{H}(G)$. Since $U C B(\hat{G}) \cap V N_{H}(G)=$ $\Phi[U C B(\hat{H})]$, there exists an $S \in U C B(\hat{H})$ such that $T=\Phi(S)$. Thus,

$$
\langle m, T\rangle=\left\langle\Phi^{*}(m), S\right\rangle=\left\langle m_{H}, S\right\rangle=\left\langle\pi_{H}\left(\theta_{H}\right), S\right\rangle=\left\langle\pi_{H}\left(\left.\theta\right|_{H}\right), S\right\rangle .
$$

On the other hand, note that $\Phi(v \cdot P)=t_{H}(v) \cdot r_{H}^{*}(P)$ for all $v \in A(H)$ and $P \in V N(H)$. Hence, we have

$$
\begin{aligned}
\left\langle\Phi^{*}(\pi(\theta)), v \cdot P\right\rangle & =\left\langle\pi(\theta), t_{H}(v) \cdot r_{H}^{*}(P)\right\rangle=\left\langle r_{H}^{*}(P), t_{H}(v) \theta\right\rangle \\
& =\left\langle P,\left.\theta\right|_{H} v\right\rangle=\left\langle\pi_{H}\left(\left.\theta\right|_{H}\right), v \cdot P\right\rangle
\end{aligned}
$$

for all $v \in A(H)$ and $P \in V N(H)$, i.e., $\pi_{H}\left(\left.\theta\right|_{H}\right)=\Phi^{*}(\pi(\theta))$. It follows that

$$
\langle m, T\rangle=\left\langle\pi_{H}\left(\left.\theta\right|_{H}\right), S\right\rangle=\left\langle\Phi^{*}(\pi(\theta)), S\right\rangle=\langle\pi(\theta), \Phi(S)\rangle=\langle\pi(\theta), T\rangle .
$$

Therefore, $m=\pi(\theta) \in B_{\rho}(G)$.

Immediately, we have the following

Theorem 3.4. Let $G$ be a locally compact group and let $\mathcal{H}_{0}$ be the collection of $\sigma$-compact open subgroups of $G$. Then $Z\left(U C B(\hat{G})^{*}\right)=B_{\rho}(G)$ if and only if $Z\left(U C B(\hat{H})^{*}\right)=B_{\rho}(H)$ for all $H \in \mathcal{H}_{0}$. 
Proof. Assume that $Z\left(U C B(\hat{G})^{*}\right)=B_{\rho}(G)$. Let $H$ be any open subgroup of $G$ and let $\Phi^{*}: U C B(\hat{G})^{*} \longrightarrow U C B(\hat{H})^{*}$ be the algebraic homomorphism as defined in Section 2. It is readily seen that for all $\varphi \in B_{\rho}(G), \Phi^{*}(\varphi)=\left.\varphi\right|_{H} \in B_{\rho}(H)$. By Lemma 2.3(ii), we have $Z\left(U C B(\hat{H})^{*}\right)=\Phi^{*}\left(Z\left(U C B(\hat{G})^{*}\right)\right)=\Phi^{*}\left(B_{\rho}(G)\right) \subseteq$ $B_{\rho}(H)$, i.e., $Z\left(U C B(\hat{H})^{*}\right)=B_{\rho}(H)$.

Conversely, suppose $Z\left(U C B(\hat{H})^{*}\right)=B_{\rho}(H)$ for all $H \in \mathcal{H}_{0}$. To get the nontrivial inclusion $Z\left(U C B(\hat{G})^{*}\right) \subseteq B_{\rho}(G)$, let $m \in Z\left(U C B(\hat{G})^{*}\right)$. Then, for all $H \in \mathcal{H}_{0}$, by Lemma 2.3(ii), $m_{H}=\Phi^{*}(m) \in Z\left(U C B(\hat{H})^{*}\right)=B_{\rho}(H)$. It follows from Lemma 3.3 that $m \in B_{\rho}(G)$.

Remark 3.5. (I) Theorem 3.4 would be trivial if we had $\overline{\bigcup_{H \in \mathcal{H}_{0}} Z\left(U C B(\hat{H})^{*}\right)^{\|\cdot\|}}=$ $Z\left(U C B(\hat{G})^{*}\right)\left(\right.$ cf. Lemma 2.3(i)). However, even though $\bigcup_{H \in \mathcal{H}_{0}} U C B(\hat{H})=U C B(\hat{G})$, $\bigcup_{H \in \mathcal{H}_{0}} Z\left(U C B(\hat{H})^{*}\right)^{\|\cdot\|}$ is in general a proper subspace of $Z\left(U C B(\hat{G})^{*}\right)$ (e.g., it is the case when $G$ is abelian but non- $\sigma$-compact).

(II) We note that Lemma 3.3 (and hence Theorem 3.4) stays true if $\mathcal{H}_{0}$ is replaced by the class $\mathcal{H}_{c}$ of compactly generated open subgroups of $G$. In fact, that $\theta$ is in $B_{\rho}(G)$ follows from the boundedness of the family $\left\{\left\|\theta_{H}\right\|_{B_{\rho}(H)}: H \in \mathcal{H}_{c}\right\}$ and the same argument as in the second paragraph of the proof of Lemma 3.2.

Now, we turn our attention to the Fourier algebra $A(G)$. We investigate whether parallel results hold for $A(G)$ and $Z\left(A(G)^{* *}\right)$.

Note that $\|u\|_{B(H)} \leq\left\|u^{\circ}\right\|_{B(G)}$ for $u \in B(H)$, where $H$ is any open subgroup of $G$ and $u^{\circ}$ is the trivial extension of $u$ to $G$ (cf. [8, Lemma 3.1]). So, one can see that Lemma 3.2 remains valid if $B_{\rho}(H)$ and $B_{\rho}(G)$ are replaced by $B(H)$ and $B(G)$, respectively. The assertion also holds if $B_{\rho}(H)$ and $B_{\rho}(G)$ are replaced by $A(H)$ and $A(G)$, respectively. However, the proof is different from the proof of Lemma 3.2. For the reader's convenience and our later use, we include the following lemma with a very short proof as suggested by the referee.

Lemma 3.6. Let $G$ be a locally compact group and let $u$ be a function on $G$ such that $\left.u\right|_{H} \in A(H)$ for all $H \in \mathcal{H}_{0}$. Then $u \in A(G)$.

Proof. Let $H_{0}$ be a fixed $\sigma$-compact open subgroup of $G$. Note that $1_{x H_{0}} \in B(G)$ for all $x \in G$. So, for any $x \in G$ and $H \in \mathcal{H}_{0}$ with $x H_{0} \subseteq H$, by the assumption, $u \cdot 1_{x H_{0}} \in t_{H}(A(H)) \subseteq A(G)$, where $t_{H}: A(H) \rightarrow A(G)$ is the trivial extension map. We also note that, for any countable subset $D$ of $G, D H_{0} \subseteq H$ for some $H \in \mathcal{H}_{0}$. Since $A(G) \cap C_{00}(G)$ is norm dense in $A(G)$ and $\left\|1_{x H_{0}}\right\|_{B(G)}=1$, it is readily seen that $\left\|u \cdot 1_{x H_{0}}\right\|_{A(G)}>0$ can hold for countably many cosets $x H_{0}$ only. Therefore, there exists an $H \in \mathcal{H}_{0}$ such that $\operatorname{supp} u \subseteq H$ and hence $u \in t_{H}(A(H)) \subseteq A(G)$.

Remark 3.7. Let $B_{\rho}(G)=A(G) \oplus B_{\rho}^{s}(G)$ be the Lebesgue decomposition (see Kaniuth-Lau-Schlichting [13, Corollary 2.5]). Then each $u \in B_{\rho}(G)$ can be written as $u=u^{a}+u^{s}$ with $u^{a} \in A(G)$ and $u^{s} \in B_{\rho}^{s}(G)$. It follows from Lemma 3.6 that

if $u \in B_{\rho}^{s}(G)$ and $u \neq 0$, then there exists an $H \in \mathcal{H}_{0}$ such that $\left(\left.u\right|_{H}\right)^{s} \neq 0$.

Our original proof of Lemma 3.6 was derived from the above assertion. 
Note that $\bigcup_{H \in \mathcal{H}_{0}} V N_{H}(G)\|\cdot\|=\bigcup_{H \in \mathcal{H}_{0}} V N_{H}(G)$. As is shown in 10, Proposition 7.3], we can have $\bigcup_{H \in \mathcal{H}_{0}} V N_{H}(G) \neq V N(G)$ when $G$ is non- $\sigma$-compact (and even abelian). Thus, we do not have the corresponding result for $A(G)^{* *}$ parallel to Lemma 3.3, i.e., for an $m \in A(G)^{* *}$, we can have $m \notin A(G)$ even $r_{H}^{* *}(m) \in A(H)$ for all $H \in \mathcal{H}_{0}$. The following proposition tells us that for such elements $m \in A(G)^{* *}$, we should take $U C B(\hat{G})^{\perp}$ (the annihilator of $U C B(\hat{G})$ in $\left.V N(G)^{*}\right)$ into account. Note that $U C B(\hat{G})=\bigcup_{H \in \mathcal{H}_{0}} r_{H}^{*}(U C B(\hat{H}))$. Therefore, for any $m \in A(G)^{* *}$,

$$
m \in U C B(\hat{G})^{\perp} \text { iff } r_{H}^{* *}(m) \in U C B(\hat{H})^{\perp} \text { for all } H \in \mathcal{H}_{0} .
$$

Clearly, $A(G) \cap U C B(\hat{G})^{\perp}=\{0\}$. We show next how $A(G) \oplus U C B(\hat{G})^{\perp}$ is related to the family $\left\{A(H) \oplus U C B(\hat{H})^{\perp}: H \in \mathcal{H}_{0}\right\}$.

Proposition 3.8. Let $G$ be a locally compact group and let $m \in A(G)^{* *}$. Then

$$
m \in A(G) \oplus U C B(\hat{G})^{\perp} \text { iff } r_{H}^{* *}(m) \in A(H) \oplus U C B(\hat{H})^{\perp} \text { for all } H \in \mathcal{H}_{0} .
$$

Proof. Obviously, if $m \in A(G) \oplus U C B(\hat{G})^{\perp}$, then $r_{H}^{* *}(m) \in A(H) \oplus U C B(\hat{H})^{\perp}$ for all open subgroups $H$ of $G$. Conversely, suppose $r_{H}^{* *}(m) \in A(H) \oplus U C B(\hat{H})^{\perp}$ for all $H \in \mathcal{H}_{0}$. Then, for each $H \in \mathcal{H}_{0}$, there exists $u_{H} \in A(H)$ such that $r_{H}^{* *}(m)-u_{H} \in U C B(\hat{H})^{\perp}$. Let $u(x)=\left\langle m, \lambda_{G}(x)\right\rangle(x \in G)$. It is evident that $\left.u\right|_{H}=u_{H} \in A(H)$ for all $H \in \mathcal{H}_{0}$. By Lemma 3.6, $u \in A(G)$. Since

$$
r_{H}^{* *}(m-u)=r_{H}^{* *}(m)-r_{H}(u)=r_{H}^{* *}(m)-u_{H} \in U C B(\hat{H})^{\perp}
$$

for all $H \in \mathcal{H}_{0}, m-u \in U C B(\hat{G})^{\perp}$. So, $m=u+(m-u) \in A(G) \oplus U C B(\hat{G})^{\perp}$.

Corollary 3.1 together with the equality $A(G) \cap U C B(\hat{G})^{\perp}=\{0\}$ implies that

if $Z\left(A(G)^{* *}\right)=A(G)$, then $\operatorname{span}\left[V N(G)^{*} V N(G)\right]$ is norm dense in $V N(G)$.

The counterexample $G=S U(3)$ by Losert shows that the converse of the above assertion is not true. In general, for non- $\sigma$-compact and non-metrizable locally compact groups (cf. Theorem 3.12), we have the following

Theorem 3.9. Let $G$ be a locally compact group and let $\mathcal{H}_{0}$ be the collection of $\sigma$-compact open subgroups of $G$. Then the following statements are equivalent:

(i) $Z\left(A(G)^{* *}\right)=A(G)$.

(ii) ${\overline{\operatorname{span}\left[V N(G)^{*} V N(G)\right]}}^{\|\cdot\|}=V N(G)$ and $Z\left(A(H)^{* *}\right)=A(H)$ for all $H \in \mathcal{H}_{0}$.

Proof. It is easy to see that if $Z\left(A(G)^{* *}\right)=A(G)$, then $Z\left(A(H)^{* *}\right)=A(H)$ for all open subgroups $H$ of $G$ (cf. the proof of [11, Proposition 8.2]). So, we only need to prove that (ii) $\Longrightarrow$ (i). Assume that $\operatorname{span}\left[V N(G)^{*} V N(G)\right]$ is norm dense in $V N(G)$ and $Z\left(A(H)^{* *}\right)=A(H)$ for all $H \in \mathcal{H}_{0}$. Let $m \in Z\left(A(G)^{* *}\right)$. Then $r_{H}^{* *}(m) \in$ $Z\left(A(H)^{* *}\right)=A(H)$ for all $H \in \mathcal{H}_{0}$. By Proposition 3.8, there exists $u \in A(G)$ such that $m-u \in U C B(\hat{G})^{\perp}$. Note that we also have $m-u \in Z\left(A(G)^{* *}\right)$. It follows from Corollary 3.1(ii) that $m-u \in Z\left(A(G)^{* *}\right) \cap U C B(\hat{G})^{\perp}=\left[V N(G)^{*} V N(G)\right]^{\perp}=\{0\}$, i.e., $m=u \in A(G)$.

If $G$ is amenable, then $B_{\rho}(G) \cdot V N(G)=V N(G)$ and hence, by Corollary 3.1(i), $\left[V N(G)^{*} V N(G)\right]^{\perp} \subseteq\left[B_{\rho}(G) \cdot V N(G)\right]^{\perp}=\{0\}$, i.e., $\operatorname{span}\left[V N(G)^{*} V N(G)\right]$ is norm dense in $V N(G)$. Immediately, we have the following corollary. 
Corollary 3.10. Let $G$ be an amenable locally compact group. Then $Z\left(A(G)^{* *}\right)=$ $A(G)$ if and only if $Z\left(A(H)^{* *}\right)=A(H)$ for all $H \in \mathcal{H}_{0}$.

We should point out that Corollary 3.10 can also be derived from Lau-Losert [17, Lemma 6.3]. For a discrete group $G$ containing the free group $F_{r}$ on $r$ generators $(2 \leq r<\infty)$, more than obtaining $Z\left(A(G)^{* *}\right) \neq A(G)$, Losert [19] actually showed that $\operatorname{span}\left[V N(G)^{*} V N(G)\right]\left(=\operatorname{span}\left[B_{\rho}(G) \cdot V N(G)\right]\right)$ is not norm dense in $V N(G)$ (cf. [19, Corollary 1]). We do not know if this is true for all non-amenable locally compact groups. Opposite to the case $\left.\overline{\operatorname{span}\left[V N(G)^{*} V N(G)\right]}\right]^{\|\cdot\|}=V N(G)$, Ülger 20. Theorem 3.3] implies that if $G$ is discrete, then $\overline{\operatorname{span}\left[V N(G)^{*} V N(G)\right]}{ }^{\|\cdot\|}=$ $U C B(\hat{G})$ if and only if $A(G)$ is Arens regular.

As is shown in [11, the assertion in Corollary 3.10 remains valid for all locally compact groups $G$ if $\mathcal{H}_{0}$ is replaced by the following family $\mathcal{H}$ :

$$
\mathcal{H}=\left\{H: H \text { is an open subgroup of } G \text { and } \kappa(H) \leq \chi(G) \cdot \aleph_{0}\right\},
$$

where $\kappa(H)$ is the compact covering number of $H$ and $\chi(G)$ is the character of $G$ (i.e., the least cardinality of an open basis at the identity of $G$ ). In fact, we have the following analogue of Lemma 3.3 with $\mathcal{H}_{0}$ replaced by $\mathcal{H}$. The proof included here is different from that given in [11, where a higher level Mazur property of $A(G)$ is used.

Lemma 3.11 ([11, Proposition 8.2]). Let $G$ be a locally compact group and let $m \in A(G)^{* *}$ be such that $r_{H}^{* *}(m) \in A(H)$ for all $H \in \mathcal{H}$. Then $m \in A(G)$.

Proof. Following the proof of Proposition 3.8 and noting that $\mathcal{H}_{0} \subseteq \mathcal{H}$, we see that there exists $u \in A(G)$ such that $r_{H}^{* *}(m)=r_{H}(u)$ for all $H \in \mathcal{H}$. We only need to show that $\langle m, T\rangle=\langle u, T\rangle$ for all $T \in V N(G)$. Let $T \in V N(G)$. Then there exists an $H \in \mathcal{H}$ such that $\operatorname{supp} T \subseteq H$ (cf. [9, Proposition 4.1]), i.e., $T \in V N_{H}(G)$. Since $V N_{H}(G)=r_{H}^{*}(V N(H)), T=r_{H}^{*}\left(T_{1}\right)$ for some $T_{1} \in V N(G)$. It follows that

$$
\langle m, T\rangle=\left\langle r_{H}^{* *}(m), T_{1}\right\rangle=\left\langle r_{H}(u), T_{1}\right\rangle=\left\langle u, r_{H}^{*}\left(T_{1}\right)\right\rangle=\langle u, T\rangle .
$$

Therefore, $m=u \in A(G)$.

Consequently, we arrive at the following

Theorem 3.12 ([11, Theorem 8.3]). Let $G$ be a locally compact group and let $\mathcal{H}$ be the collection of open subgroups of $G$ as defined above. Then $Z\left(A(G)^{* *}\right)=A(G)$ if and only if $Z\left(A(H)^{* *}\right)=A(H)$ for all $H \in \mathcal{H}$.

In particular, if $G$ is a metrizable locally compact group, then $Z\left(A(G)^{* *}\right)=A(G)$ if and only if $Z\left(A(H)^{* *}\right)=A(H)$ for all $H \in \mathcal{H}_{0}$.

Remark 3.13. (I) By [9, Proposition 4.1], we have $\left.\overline{\operatorname{span}\left[V N(G)^{*} V N(G)\right]}\right]^{\|\cdot\|}=$ $V N(G)$ if and only if $\left.\overline{\operatorname{span}\left[V N(H)^{*} V N(H)\right]}\right]^{\|\cdot\|}=V N(H)$ for all $H \in \mathcal{H}$. Thus, Theorem 3.12 also follows from Theorem 3.9.

(II) Note that $\left[B_{\rho}(G) \cdot V N(G)\right]^{\perp} \subseteq U C B(\hat{G})^{\perp}$. Comparing with Proposition 3.8 , it is natural to ask whether for all $m \in A(G)^{* *}$, we have

$$
\begin{aligned}
& m \in A(G) \oplus\left[B_{\rho}(G) \cdot V N(G)\right]^{\perp} \text { iff } r_{H}^{* *}(m) \in A(H) \oplus\left[B_{\rho}(H) \cdot V N(H)\right]^{\perp} \\
& \text { for all } H \in \mathcal{H}_{0} .
\end{aligned}
$$

By Proposition 3.8 and Corollary 3.1(ii), it is readily seen that (1) holds for all $m \in Z\left(A(G)^{* *}\right)$. However, (1) does not hold in general for all $m \in A(G)^{* *}$ (see 
the paragraph following Remark 3.7). On the other hand, owing to 9, Proposition 4.1], we have $B_{\rho}(G) \cdot V N(G)=\bigcup_{H \in \mathcal{H}} B_{\rho}(H) \cdot V N(H)$. Therefore, (1) remains valid if $\mathcal{H}_{0}$ is replaced by $\mathcal{H}$, i.e., for all $m \in A(G)^{* *}$, we have

(2) $m \in A(G) \oplus\left[B_{\rho}(G) \cdot V N(G)\right]^{\perp}$ iff $r_{H}^{* *}(m) \in A(H) \oplus\left[B_{\rho}(H) \cdot V N(H)\right]^{\perp}$ for all $H \in \mathcal{H}$.

The above arguments are also valid when $B_{\rho}(G)$ (resp. $\left.B_{\rho}(H)\right)$ is replaced by $V N(G)^{*}\left(\operatorname{resp} . V N(H)^{*}\right)$.

Lau-Losert [17, Theorem 5.8] showed that if $G$ is second countable and $\overline{[G, G]}$ is not open in $G$, then $Z\left(U C B(\hat{G})^{*}\right)=B_{\rho}(G)$. They proved in the same paper that if $G$ is amenable, and $Z\left(U C B(\hat{G})^{*}\right)=B_{\rho}(G)$, then $Z\left(A(G)^{* *}\right)=A(G)$ (cf. 17, Theorem 6.4]). Therefore, $Z\left(A(G)^{* *}\right)=A(G)$ if $G$ is second countable and amenable, and $\overline{[G, G]}$ is not open in $G$ ([17, Theorem 6.5(iii)]). Applying Theorem 3.4, we have the following minor extension of [17, Theorem 5.8 and Theorem 6.5(iii)].

Theorem 3.14. Let $G$ be a metrizable locally compact group such that $\overline{[G, G]}$ is not open in $G$. Then

(i) $Z\left(U C B(\hat{G})^{*}\right)=B_{\rho}(G)$.

(ii) $Z\left(A(G)^{* *}\right)=A(G)$ if $G$ is amenable.

Proof. (i) Let $H$ be any $\sigma$-compact open subgroup of $G$. Then $H$ is a second countable locally compact group. Note that $\overline{[H, H]}$ is not open in $H$ since $\overline{[H, H]} \subseteq$ $\overline{[G, G]}$, and $\overline{[G, G]}$ is not open in $G$. By [17, Theorem 5.8], $Z\left(U C B(\hat{H})^{*}\right)=B_{\rho}(H)$. So, by Theorem $3.4, Z\left(U C B(\hat{G})^{*}\right)=B_{\rho}(G)$.

(ii) It follows from (i) and [17, Theorem 6.4].

Corollary 3.15. Let $G=\prod_{n} G_{n}$ be a finite or countable product of metrizable locally compact groups such that $G_{n}$ is compact for all but finitely many n. Assume that either $\overline{\left[G_{1}, G_{1}\right]}$ is not open in $G_{1}$ or $G_{1}$ is abelian and non-discrete. Then

(i) $Z\left(U C B(\hat{G})^{*}\right)=B_{\rho}(G)$.

(ii) $Z\left(A(G)^{* *}\right)=A(G)$ if all $G_{n}$ are amenable.

Proof. Clearly, $G$ is a metrizable locally compact group. According to Theorem 3.14, we only need to show that $\overline{[G, G]}$ is not open in $G$.

Let $q: G \longrightarrow G_{1}$ be the canonical projection. Then $q([G, G]) \subseteq\left[G_{1}, G_{1}\right]$ and hence $q(\overline{[G, G]}) \subseteq \overline{q([G, G])} \subseteq \overline{\left[G_{1}, G_{1}\right]}$. Since $q: G \longrightarrow G_{1}$ is an open map, $\overline{[G, G]}$ is not open in $G$ if $\overline{\left[G_{1}, G_{1}\right]}$ is not open in $G_{1}$.

Assume now $G_{1}$ is abelian and non-discrete. Let $G^{\prime}=\prod_{n \neq 1} G_{n}$. Then $\overline{[G, G]} \subseteq G^{\prime}$. Since $G_{1} \cong G / G^{\prime}$ is non-discrete, $G^{\prime}$ is not open in $G$ and hence $\overline{[G, G]}$ is not open in $G$.

A close inspection of the proof of Lemma 3.2, Lemma 3.3 and Theorem 3.4 shows that Lau-Losert [18, Theorem 4.2] is still true if the group $G_{0}$ is assumed to be metrizable (but may not be second countable). More precisely, for the group $G=G_{0} \times \prod_{i=1}^{\infty} G_{i}$ as in [18, Theorem 4.2] with $G_{0}$ only metrizable, our Lemma 3.2, 
Lemma 3.3 and hence Theorem 3.4 remain valid if $\mathcal{H}_{0}$ is replaced by the family

$$
\left\{H \times \prod_{i=1}^{\infty} G_{i} \mid H \text { is a } \sigma \text {-compact open subgroup of } G_{0}\right\} .
$$

Therefore, slightly extending Lau-Losert [18, Theorem 4.2], we have the following

Theorem 3.16. Let $G=G_{0} \times \prod_{i=1}^{\infty} G_{i}$, where each $G_{i}(i \geq 0)$ is a metrizable locally compact group and $G_{i}$ is compact and non-trivial for $i \geq 1$. Then

(i) $Z\left(U C B(\hat{G})^{*}\right)=B_{\rho}(G)$.

(ii) $Z\left(A(G)^{* *}\right)=A(G)$ if $G_{0}$ is amenable.

\section{ACKNOWLEDGEMENT}

The author is indebted to the referee for the valuable comments, in particular, for suggesting Remark 3.5(II) and the current short proof of Lemma 3.6.

\section{REFERENCES}

1. R. Arens, The adjoint of a bilinear operation, Proc. Amer. Math. Soc. 2 (1951), 839-848. MR.0045941 (13:659f)

2. P. Eymard, L'algèbra de Fourier d'un groupe localement compact, Bull. Soc. Math. France 92 (1964), 181-236. MR0228628 (37:4208)

3. E. E. Granirer, Weakly almost periodic and uniformly continuous functionals on the Fourier algebra of any locally compact group, Trans. Amer. Math. Soc. 189 (1974), 371-382. MR0336241 (49:1017)

4. E. E. Granirer, Density theorems for some linear subspaces and some $C^{*}$-algebras of $V N(G)$, Istituto Nazionale di Alta Mathematica, Symposia Mathematica 22 (1977), 61-70. MR0487287 (58:6935)

5. M. Grosser and V. Losert, The norm-strict bidual of a Banach algebra and the dual of $C_{u}(G)$, Manuscript Math. 45 (1984), 127-146. MR0724731(86b:46073)

6. C. Herz, Harmonic synthesis for subgroups, Annales de l'Institut Fourier (Grenoble) 23 (1973), 91-123. MR0355482(50:7956)

7. E. Hewitt and K. A. Ross, Abstract Harmonic Analysis I, Springer-Verlag, New York, 1979. MR0551496 (81k:43001)

8. Z. Hu, Open subgroups of $G$ and almost periodic functionals on $A(G)$, Proc. Amer. Math. Soc. 128 (2000), 2473-2478. MR.1662249 (2000k:22009)

9. Z. Hu, Inductive extreme non-Arens regularity of the Fourier algebra $A(G)$, Studia Math. 151 (2002), 247-264. MR1917836 (2003f:46069)

10. Z. Hu, Maximally decomposable von Neumann algebras on locally compact groups and duality, Houston J. Math. 31 (2005), 857-881. MR2148807

11. Z. Hu and M. Neufang, Decomposability of von Neumann algebras and Mazur property of higher level, Canad. J. Math, to appear.

12. N. Isik, J. S. Pym and A. Ülger, The second dual of the group algebra of a compact group, J. London Math. Soc. (2) 35 (1987), 135-148. MR0871771 (88f:43012)

13. E. Kaniuth, A. T. Lau and G. Schlichting, Lebesgue type decomposition of subspaces of Fourier-Stieltjes algebras, Trans. Amer. Math. Soc. 355 (2003), 1467-1490. MR.1946400 (2004c:43004)

14. A. T. Lau, Uniformly continuous functionals of the Fourier algebra of any locally compact group, Trans. Amer. Math. Soc. 251 (1979), 39-59. MR0531968 (80m:43009)

15. A. T. Lau, Continuity of Arens multiplication on the dual space of bounded uniformly continuous functions on locally compact groups and topological semigroups, Math. Proc. Cambridge Philos. Soc. 99 (1986), 273-283. MR0817669 (87i:43001)

16. A. T. Lau and V. Losert, On the second conjugate algebra of a locally compact group, J. London Math. Soc. 37 (1988), 464-470. MR0939122 (89e:43007)

17. A. T. Lau and V. Losert, The $C^{*}$-algebra generated by operators with compact support on a locally compact group, J. Funct. Anal. 112 (1993), 1-30. MR.1207935 (94d:22005) 
18. A. T. Lau and V. Losert, The centre of the second conjugate algebra of the Fourier algebra for infinite product of groups, Math. Proc. Cambridge Philos. Soc., 138 (2005), 27-39. MR2127225 (2006c:43003)

19. V. Losert, The centre of the bidual of Fourier algebras (discrete groups), preprint.

20. A. Ülger, Central elements of $A^{* *}$ for certain Banach algebras A without bounded approximate identities, Glasgow Math. J. 41(1999), 369-377. MR.1720442 (2001b:46082)

Department of Mathematics and Statistics, University of Windsor, Windsor, OnTARIO, CANADA N9B 3P4

E-mail address: zhiguohu@uwindsor.ca 